EPJ Web of Conferences 32, 01005 (2012)

DOI: $10.1051 /$ epjconf/20123201005

(C) Owned by the authors, published by EDP Sciences, 2012

\title{
Assessment of Electron-Cyclotron-Current-Drive-Assisted Operation in DEMO
}

\author{
E. Poli ${ }^{1, \text { a }}$, E. Fable ${ }^{1}$, G. Tardini ${ }^{1}$, H. Zohm ${ }^{1}$, D. Farina ${ }^{2}$, L. Figini ${ }^{2}$, N. B. Marushchenko ${ }^{3}$, and \\ L. Porte ${ }^{4}$ \\ 1 Max-Planck-Institut für Plasmaphysik, Garching bei München, Germany \\ 2 Istituto di Fisica del Plasma CNR, EURATOM-ENEA-CNR Association, Milano, Italy \\ 3 Max-Planck-Institut für Plasmaphysik, EURATOM Association, Teilinstitut Greifswald, Germany \\ ${ }^{4}$ Centre de Recherches en Physique des Plasmas, CRPP-EPFL, Lausanne, Switzerland
}

\begin{abstract}
The achievable efficiency for external current drive through electron-cyclotron (EC) waves in a demonstration tokamak reactor is discussed. Two possible reactor designs, one for steady state and one for pulsed operation, are considered. It is found that for midplane injection the achievable current drive efficiency is limited by secondharmonic absorption at levels consistent with previous studies. Propagation through the second-harmonic region can be reduced by moving the launch position to the high-field side (this can be obtained by injecting the beam from an upper port in the vacuum vessel). In this case, beam tracing calculations deliver values for the EC current drive efficiency approaching those usually reported for neutral beam current drive.
\end{abstract}

\section{Introduction}

In a fusion reactor based on the tokamak concept, the fraction of non-inductive current should be as high as possible to approach steady-state operation. For this reason, current-drive methods with high efficiency (large current per unit injected power) should be devised. Electron cyclotron current drive (ECCD) [1-3] is one of the possible candidates. From the technological point of view, ECCD is considered the most mature among the heating and CD systems envisaged for ITER, due to the availability of reliable high-power sources and to the simplicity of the wave-plasma coupling. However, the CD efficiency of EC waves is usually considered too poor as compared, for instance, with neutral beam injection (NBI). In fact, ECCD alone is not considered as an option for ITER steady-state scenarios within the present design [4]. On the other hand, it should be considered that present ECRH systems (including ITER) have not been designed with the main goal of maximizing the amount of driven current. Moreover, in addition to the CD efficiency, in a reactor one should also consider the wall-plug efficiency of the system, i. e. its efficiency in converting electrical power into injected power. Finally, as the number and size of apertures in the vessel of a reactor should be reduced as much as possible, ECCD offers a clear structural advantage with respect to NBI.

The main goal of this paper is to explore the achievable CD efficiency for two selected options for a demonstration power plant (DEMO). One DEMO design should be operated under steady-state conditions, therefore is external current drive particularly critical in this case. The second design is based on the assumption of pulsed operation (6-hours-discharges with 15-minutes-downtime in between). The machine and plasma parameters for both options are given below in the paper. In reactor studies, the $\mathrm{CD}$ efficiency is often specified in terms of the figure

$$
\gamma_{C D}=\frac{n_{20} R_{\mathrm{m}} I_{\mathrm{A}}}{P_{\mathrm{W}}}
$$

\footnotetext{
a e-mail: Emanuele.Poli@ipp.mpg.de
}

This is an Open Access article distributed under the terms of the Creative Commons Attribution License 2.0, which permits unrestricted use, distribution, and reproduction in any medium, provided the original work is properly cited. 
where $n_{20}$ is the density expressed in units of $10^{20}$ particles per cubic meter, $R_{\mathrm{m}}$ is the major radius of the machine in metres, $I_{\mathrm{A}}$ the driven current in amperes, and $P_{\mathrm{W}}$ the injected power in watts. For ITER, $\gamma_{C D}$ is usually estimated to be around 0.2 and 0.3 for ECCD and NBCD, respectively [4]. A recent DEMO study found values again around 0.2 for ECCD and values between 0.45 and 0.55 for NBCD depending on the acceleration voltage [5].

\section{DEMO Parameters}

The first set of DEMO parameters used in ECCD calculations corresponds to a machine operating steady-state, with major radius $R_{0}=8.5 \mathrm{~m}$, minor radius $a=2.83 \mathrm{~m}$, on-axis magnetic field $B=5.84$ $\mathrm{T}$, normalized pressure $\beta_{N}=2.95$. Both flat and peaked density profiles are considered, as recent theoretical studies predict density peaking with decreasing collisionality, even in the presence of central EC heating [6,7]. Density and temperature profiles are shown in Fig. 1.
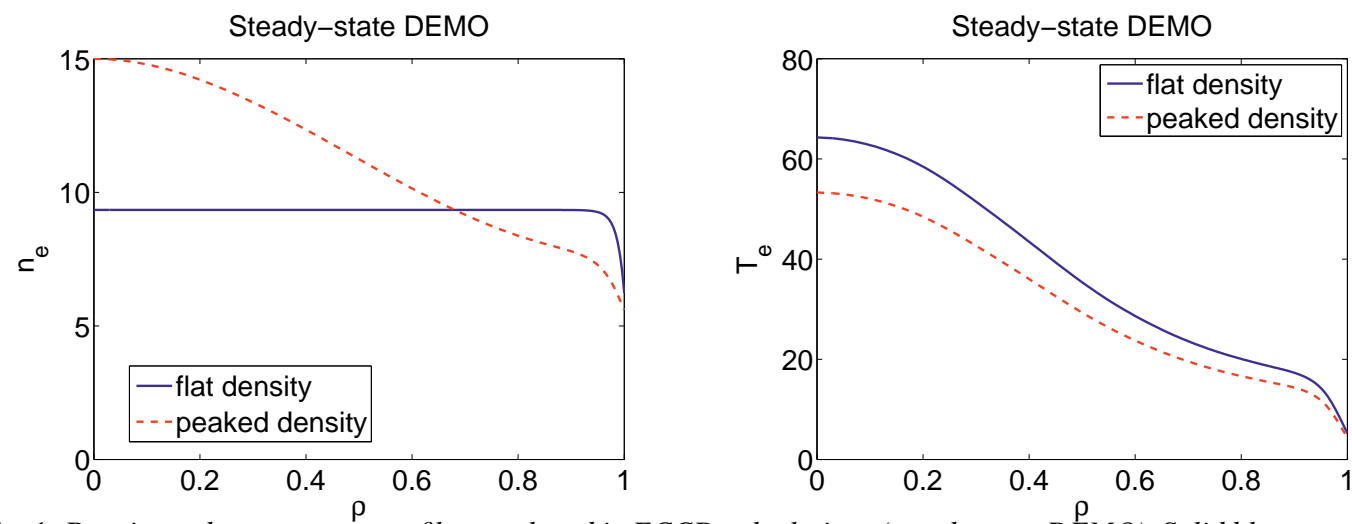

Fig. 1. Density and temperature profiles employed in ECCD calculations (steady-state DEMO). Solid blue curves: "flat-density" case; dashed red curves: "peaked-density" case.

The density profile at the pedestal top $\left(\rho_{p}=0.9\right)$ is taken equal to the Greenwald density, as many experimental results suggest that the Greenwald limit is an edge limit. In the flat-density case, this value is simply prolonged down to the plasma centre. In the peaked-density case, the shape of the density profile is taken according to an ASDEX Upgrade improved H-mode shot (also known as hybrid mode of operation) The plasma current, that determines the value of the Greenwald density, is chosen to be the minimum current required for ignition, $P_{\text {loss }}=P_{\text {fus }} / 5$ (an H-mode enhancement factor $H=1.2$ is assumed). The shape of the temperature profile is also taken from improved-H-mode discharges of ASDEX Upgrade, rescaled such as to obtain the targeted value of $\beta_{N}$.

The second set of machine parameters describe a pulsed device, as stated before. To achieve a substantial pulse length, a large central solenoid must be accommodated. Moreover, a larger aspect ratio favours long pulse durations. For this reason, the pulsed DEMO considered here is a larger machine with $R_{0}=9.6 \mathrm{~m}, a=2.4 \mathrm{~m}, B=7.45 \mathrm{~T}, \beta_{N}=2.6$. Density and temperature profiles are chosen according to the same prescription as for the steady-state case.

\section{Optimization of the ECCD Efficiency}

For the evaluation of ECCD for the DEMO plasmas described above, the beam tracing code TORBEAM [8] is employed. Diffraction effects on the beam propagation are described through the paraxial approximation [9]. For the modelling of absorption and current drive, the fully-relativistic absorption routines developed for the codes GRAY [10] and TORAY [11] can be used, along with Lin-Liu's current drive routine [12], augmented with a procedure to ensure momentum conservation in electronelectron collisions [13] first developed for the TRAVIS code. The value of the effective charge is $Z_{\text {eff }}=2.57$ for the steady-state DEMO and $Z_{\text {eff }}=1.95$ for the pulsed DEMO. 
The very high temperatures of DEMO plasmas lead to different CD conditions as compared to present-day tokamaks (similar conditions, however, are approached in simulations for ITER advanced scenarios [14] and have been considered also in previous studies for the old ITER design [15]). For instance, maximum current drive on a given flux surface can be usually obtained by setting the resonance on the high-field side, since this minimizes the impact of trapped particles. For this reason, it is beneficial to choose a high wave frequency, thus setting the resonance layer at smaller major radii. However, this implies that the wave travels longer in the region where no first-harmonic absorption is possible. For DEMO parameters, parasitic second-harmonic absorption, reducing the power available for current drive at the desired position, starts to be significant when the peak first-harmonic absorption is still on the low-field side of the magnetic axis, so that high-field-side CD turns out to be very challenging.

From the resonance condition

$$
\frac{n \Omega}{\omega}=\gamma-N_{\|} u_{\|}
$$

( $n$ is the harmonic number, $\Omega=e B / m_{e}$ the electron cyclotron frequency, $\omega$ the wave frequency, $\gamma=$ $\sqrt{1+u^{2}}$ the relativistic Lorentz factor and $N_{\|}$the parallel component of the refractive index vector $\mathbf{N}=$ $c \mathbf{k} / \omega)$ it is easy to show that first-harmonic absorption is possible only for $R \leq\left(\Omega_{0} / \omega \sqrt{1-N_{\|}^{2}}\right) R_{0}$, where $\Omega_{0}$ is the cyclotron frequency at the geometric axis. Fig. 2 shows the values of $R$ at which first harmonic absorption becomes accessible for different values of the wave frequency and of the parallel refractive index.
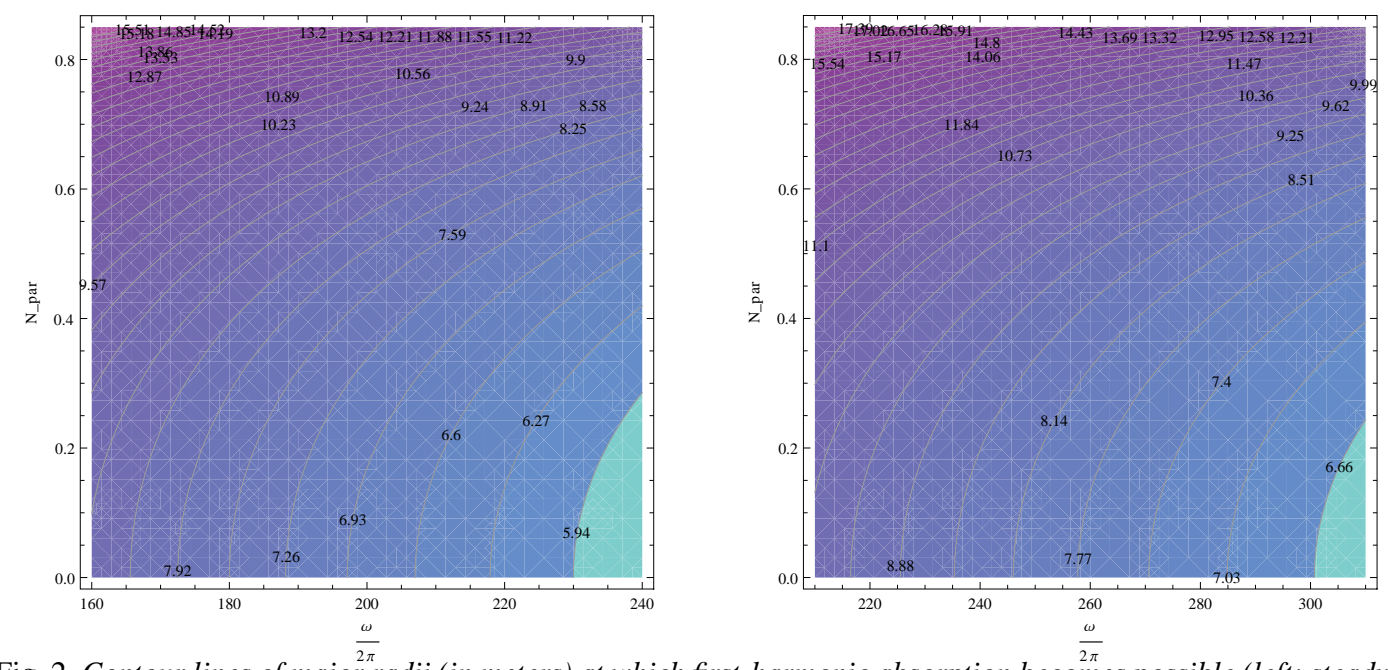

Fig. 2. Contour lines of major radii (in meters) at which first-harmonic absorption becomes possible (left: steadystate DEMO parameters; right: pulsed DEMO parameters).

A way to reduce the path through the region of parasitic absorption is thus to move the launcher location towards the high-field side. Wave injection from the midplane and from higher positions is discussed below. It should be notices that at high $N_{\|}$-values, a small change in $N_{\|}$can result in a large shift of the first-harmonic resonance.

\subsection{Steady-state DEMO}

Equatorial injection (poloidal launch angle $\alpha=0$ ) for steady-state DEMO parameters is considered first. The radial position of maximum absorption and the corresponding CD efficiency are shown in Fig. 3 for the peaked density case as a function of the wave frequency and of the toroidal angle $\beta$.

For a given toroidal angle, the driven current as a function of the wave frequency first rises as the resonance is pushed towards the plasma core and then decreases because of increasing parasitic 
absorption at the second cyclotron harmonic. The CD efficiency $\gamma_{C D}$ has a maximum around $\omega / 2 \pi=$ $215 \mathrm{GHz}$ and $\beta=40^{\circ}$, with values slightly above 0.3 for the peaked-density and around 0.27 for the flat-density case. This slightly lower efficiency of the flat-density case as compared to the peakeddensity case is due to the fact the absorption takes place at larger minor radii, where the fraction of trapped particles is higher (the flat-density case has smaller Shafranov shift, so that the resonance layer intersects the flux surfaces at larger radii). It is noted that these values for frequency, injection angle and $\gamma_{C D}$ are in line with the results reported in Ref. [15], where a saturation (due to parasitic absorption) at around $\gamma_{C D}=0.3$ for temperatures above $30 \mathrm{keV}$ and for midplane injection was observed (the steadystate DEMO parameters considered here are similar to the ITER parameters analyzed in Ref. [15]). The sensitivity of this scheme to parasitic absorption can be illustrated by increasing the frequency from 215 to $225 \mathrm{GHz}$, or decreasing the toroidal injection angle from $40^{\circ}$ to $35^{\circ}$, that leads to an increase of second-harmonic absorption from $6 \%$ of the total absorbed power to $31 \%$ and $23 \%$, respectively.
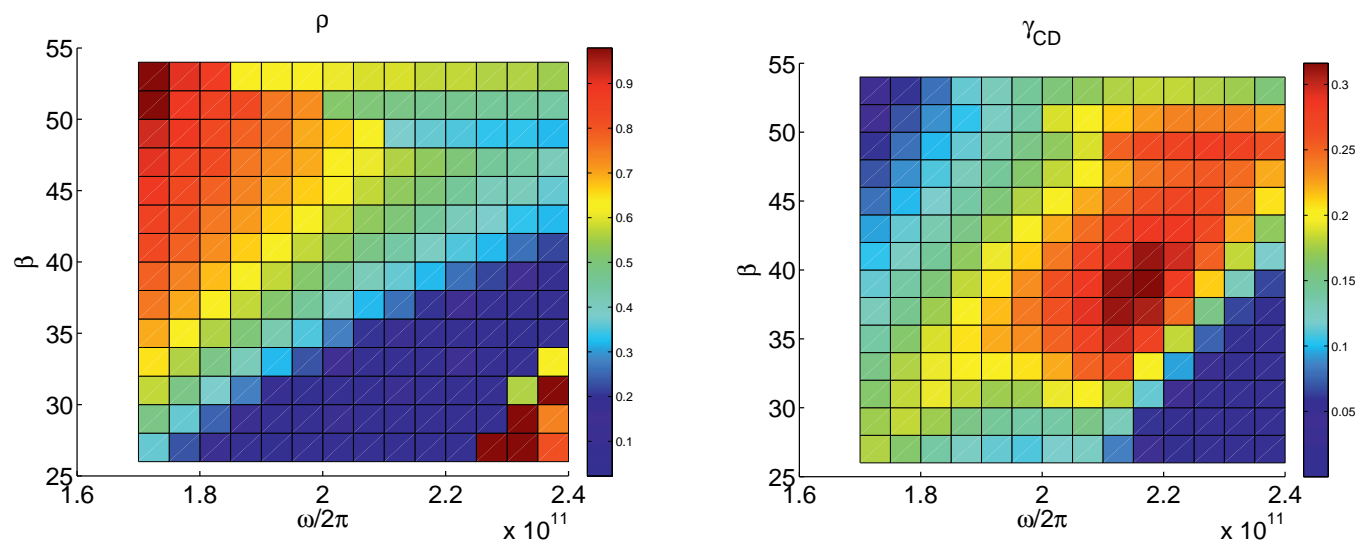

Fig. 3. Deposition radius and CD efficiency for propagation in the midplane (poloidal injection angle $\alpha=0$ ) from $(R, Z)=(12.0,0.5) m$ as a function of wave frequency and toroidal injection angle $\beta$ (steady-state DEMO, peaked density case).

The CD efficiency for a beam injected from a higher position, $(R, Z)=(10.5,3.5) \mathrm{m}$, is shown in Fig. 4. Values of $\gamma_{C D}$ close to 0.4 can be reached for $\omega / 2 \pi=230 \mathrm{GHz}$ with peak at $\rho_{p} \simeq 0.3$. To quantify the effect of momentum conservation on this result, the same scan has been repeated employing the usual high-speed-limit in the calculation of the Spitzer function. In this case, the peak current drive efficiency is reduced by $20 \%$ ( 0.32 instead of 0.4 ), as expected from previous investigations $[15,16]$.
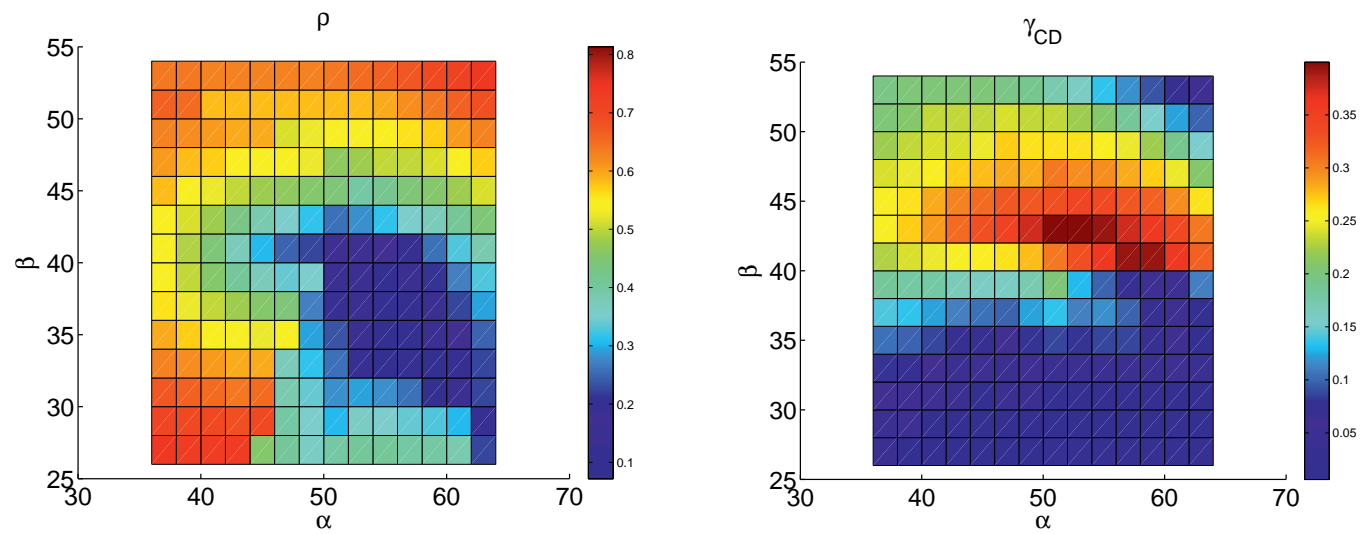

Fig. 4. Deposition radius and CD efficiency for injection from the upper part of the vessel, $(R, Z)=(10.5,3.5)$ $m$, as a function of the poloidal injection angle $\alpha$ and the toroidal injection angle $\beta$, for a wave frequency of 230 GHz (steady-state DEMO, peaked density case). 
The dependence of the maximum CD efficiency and of the corresponding deposition radius on the wave frequency for different launch locations is plotted in Fig. 5.
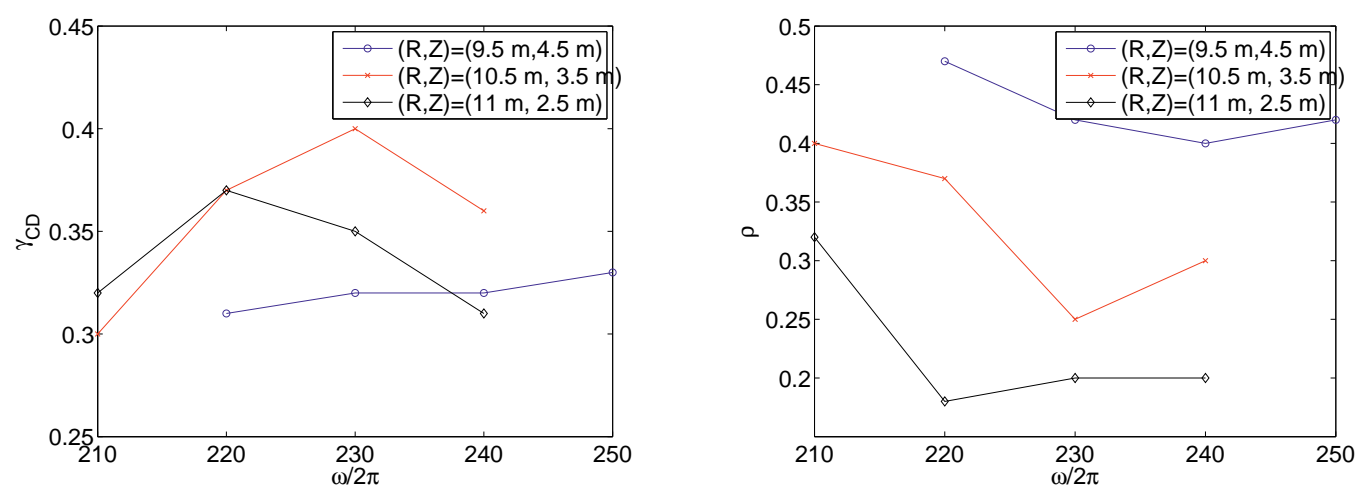

Fig. 5. Maximum CD efficiency (left) and corresponding radial location (right) as a function of the injected wave frequency for the launch positions indicated in the legend.

It can be seen that moving the launch position towards the top of the vacuum vessel leads to an outward shift of the location of maximum efficiency. Moreover, the maximum efficiency is obtained for higher and higher frequencies.

\subsection{Pulsed DEMO}

Because of the much higher magnetic field, higher frequencies must be considered for ECCD in the pulsed DEMO designed discussed here. Repeating the previous exercise, it is found that high current densities and high CD efficiencies are obtained when the launch position is moved towards the top of the machine, as shown in Fig. 6.
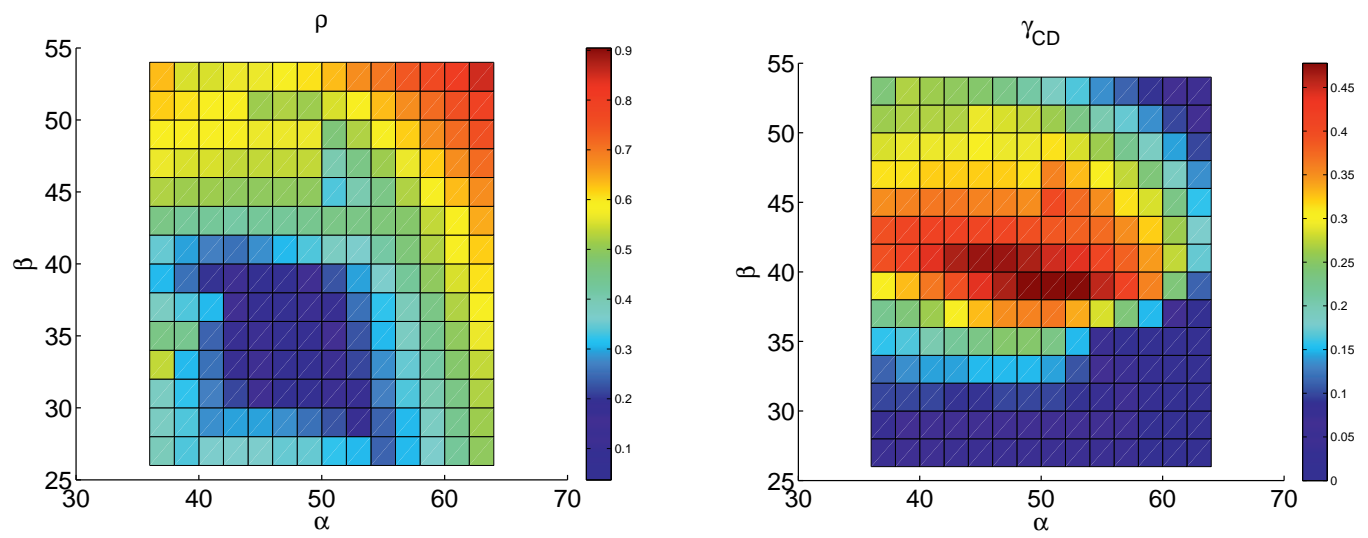

Fig. 6. Deposition radius and CD efficiency for injection from the upper part of the vessel, $(R, Z)=(12.0,3.0) \mathrm{m}$ as a function of the poloidal and toroidal injection angles, for a frequency of $290 \mathrm{GHz}$ (pulsed DEMO, peaked density case).

Because of the larger aspect ratio (smaller trapped-particle fraction) and the smaller value of $Z_{\text {eff }}$, the amount of driven current can be very high, resulting in CD efficiencies $\gamma_{C D}>0.45$. These values are attained for wave frequencies slightly below $300 \mathrm{GHz}$. 


\section{Summary}

The results presented above show that a comparatively high ECCD efficiency can be obtained in reactor-grade plasmas. The estimates for the figure $\gamma_{C D}$ are significantly higher than those commonly found in the literature, exhibiting peak values close to 0.4 (or even above this value for the parameters of a pulsed reactor considered here). The maximum efficiency is usually attained at poloidal radii around $\rho_{p} \simeq 0.3$. The high efficiency reported here is due to several factors. First of all, the EC driven current has been computed including momentum conservation, which leads to higher values (by about $20 \%$ ) as compared to the the high-speed-limit scheme usually employed in linear calculations and in systems codes. Moreover, the electron temperatures resulting from our assumptions on the density profile and on the target value for $\beta_{N}$ are somewhat higher than those considered in previous analyses (the beneficial effect on CD efficiency of operating a reactor at temperatures above the optimum temperature for fusion reactions has been pointed out previously [17]). However, to circumvent the saturation of the $\mathrm{CD}$ efficiency at high temperatures due to increasing parasitic absorption, the injection position and the frequency must be selected carefully. High CD efficiency is obtained moving the launch position towards the high-field side. This can be achieved by injecting the beams from a port in the upper part of the vessel.

The combination of linear absorption and adjoint determination of the CD efficiency offers a wellbenchmarked, reliable tool for the investigation presented in this paper. The broad absorption profiles typical of high-CD injection geometries result generally in relatively low power densities, ruling out quasi-linear effects [18] even for an injected power of several hundred MW (the centre of the plasma column, where the power density could be significantly higher, is usually not reachable and should probably be avoided anyway, in order to prevent the safety factor from dropping to too low values). An evaluation of synchrotron losses on the CD efficiency [19] is still to be addressed.

The optimization of the CD efficiency is obviously only a first step towards the design of a CD system for reactor operations. Since this system is supposed to drive a significant part of the plasma current, the effects of the injection of more than $200 \mathrm{MW}$ on a given magnetic configuration and on the corresponding kinetic profiles is to be investigated self-consistently, as done e. g. in Ref. [5]. The optimum ECCD profile depends on the kind of scenario envisaged for (nearly) steady-state discharges. This study is planned for the near future.

\section{References}

1. M. Bornatici et al., Nucl. Fusion 23 (1983), 1159

2. V. Erckmann, U. Gasparino, Plasma Phys. Control. Fusion 36(1994), 1869

3. R. Prater, Phys. Plasmas 11 (2004), 2349

4. F. Wagner et al., Plasma Phys. Control. Fus. 52 (2010), 124044

5. J. Garcia et al., Nucl. Fusion 48 (2008), 075007

6. C. Angioni et al., Phys. Rev. Lett. 90 (2003), 205003

7. G. V. Pereverzev et al., Nucl. Fusion 45 (2005), 221

8. E. Poli, A. G. Peeters, G. V. Pereverzev, Comp. Phys. Comm. 136 (2001), 90

9. G. V. Pereverzev, Phys. Plasmas 5 (1998), 3529

10. D. Farina, Fus. Sci. Tech. 53 (2008), 130

11. E. Westerhof, Implementation of Toray at JET, Rijnhuizen Report RR-89-183 (1989).

12. Y. R. Lin-Liu, V. S. Chan, R. Prater, Phys. Plasmas 10 (2003), 4064

13. N. B. Marushchenko, C. D. Beidler, H. Maassberg, Fus. Sci. Tech. 55 (2009), 180

14. D. Farina et al., Nucl. Fusion 52 (2012), 033005

15. R. W. Harvey et al., Nucl. Fusion 37 (1997), 69

16. N. B. Marushchenko, H. Maassberg, Yu. Turkin, Nucl. Fusion 48 (2008), 054002 and Erratum Nucl. Fusion 49 (2009), 129801

17. D. J. Ward, Plasma Phys. Control. Fus. 52 (2010), 124033

18. R. W. Harvey, M. G. McCoy, G. D. Kerbel, Phys. Rev. Lett. 62 (1989), 426

19. S. V. Kasilov, W. Kernbichler, Phys. Plasmas 3 (1996), 4115 